\title{
DIE RECHNUNGSHÖFE GUATEMALAS UND COSTA RICAS
}

\author{
Von Dieter OberndöRfer
}

Wie in den meisten lateinamerikanischen Staaten ist auch in Guatemala und Costa Rica eine Kontrolle der staatlichen Einnahmen und Ausgaben durch Rechnungshöfe von der Verfassung vorgesehen. Im folgenden wird untersucht, inwieweit die beiden Rechnungshöfe ihren gesetzlichen Auftrag erfüllen ${ }^{1}$. In einem abschließenden Vergleich wird auf die für ihre Leistungen konstitutiven politischen Rahmenbedingungen verwiesen.

\section{Der Rechnungshof Guatemalas (Contraloría de Cuentas)}

\section{Rechtliche Grundlagen}

Rechtsgrundlagen sind Art. 226-28 der Verfassung vom 15. September 1965, das Gesetz zur Schaffung des Rechnungshofes ${ }^{2}(=$ L. O.) vom 21. 11. 1956 und die Durchführungsverordnung (= Regl.) vom 27. 12. $1958^{3}$.

\section{Verfassungsrechtliche Stellung und Aufgaben}

Die verfassungsrechtliche Stellung und die Aufgaben des Rechnungshofes werden von der Verfassung und durch das Gesetz wie folgt bestimmt:

„Der Rechnungshof ist eine technische Institution mit absoluter Unabhängigkeit der Funktionen “". „Er überprüft die Einnahmen, die Ausgaben und die Vermögensinteressen des Staates, der Stadt (gemeint ist die Stadt Guatemala - d. Verf.), der dezentralisierten autonomen und halbautonomen staatlichen Behörden, sowie darüber hinaus jeglicher Einrichtung oder Person, die staatliche Gelder erhält oder öffentliche Sammlungen veranstaltet ${ }^{5}$."

Úber Formeln wie „technische Institution mit absoluter Unabhängigkeit der Funktionen" hinaus, wird die Stellung des Rechnungshofes innerhalb der Gewaltenteilung vom Gesetzgeber nicht weiter präzisiert, Beiträge zu diesem Fragenkomplex von seiten guatemaltekischer Rechtswissenschaftler liegen nicht vor.

\section{Die Leitung des Rechnungshofes: Der Generalkontrolleur (Contralor General)}

Zur Sicherung der Unabhängigkeit des Rechnungshofes sieht die Verfassung von 1965 im Unterschied zur früheren Praxis (Ernennung des Chefs der Behörde und

1 Der Aufsatz beruht auf einer Studie, die der Verfasser gemeinsam mit Hermann Avenarius, Ulrich Fanger und Jürgen $\mathrm{H}$. Wolff 1973 durchgeführt hat. Ergebnisse zur Finanzverwaltung wurden bereits in VRU Heft 4, 1974 und Heft 1, 1975 vorgelegt.

2 Ley Orgánica del Tribunal y Contraloría de Cuentas, Decreto 1226 del Congreso, im folgenden zitiert: L. O.

3 Reglamento de la Ley Orgánica de la Contraloría de Cuentas, im folgenden zitiert: Regl.

4 Art. 2, L. O.

4 Art. 2, L. Ó. 5 Constitución de la República de Guatemala, Decreto de la Asamblea Nacional Constituyente en 15 de

Constitución de la República de Guatemala, Decreto de la Asamblea Nacional Constituyente en 15 de
Septiembre de 1965, Art. 226. Der Verfassungstext war zur Zeit der Untersuchung (Februar-April 1973) in sämtlichen Buchhandlungen und auch in der Staatsdruckerei nicht erhältlich! 
seiner Beamten durch den Präsidenten) die Wahl des Contralor General durch den Kongreß auf eine Amtszeit von vier Jahren vor ${ }^{6}$. Sie überläßt dem Generalkontrolleur die Personalhoheit, das Recht, die Beamten und Angestellten des Rechnungshofes $\mathrm{zu}$ ernennen und $\mathrm{zu}$ entlassen ${ }^{7}$. Da es einen effektiven beamtenrechtlichen Schutz der Bediensteten gegen Entlassungen nicht gibt, wird dadurch eine ausgesprochen autoritäre oder besser autokratische Leitung möglich. Es kommt hinzu, daß die Rechnungsprüfer nur im Rahmen der Weisungen des Generalkontrolleurs tätig werden können, d. h. von Routineprüfungen abgesehen, entscheidet der Generalkontrolleur darüber, wo Prüfungen durchgeführt werden dürfen ${ }^{8}$.

Über eine eventuelle Abwahl des Generalkontrolleurs bemerkt die Verfassung lediglich: „Er kann vom Kongreß in den Fällen und in der Form, die das Gesetz vorsieht, seines Amtes enthoben werden"." Entsprechende gesetzliche Bestimmungen, die die obige Verfassungsbestimmung präzisieren, gibt es nicht. Einen Hinweis gibt vielleicht Artikel 228 der Verfassung, in der dem Generalkontrolleur die gleiche Immunität zugestanden werden, wie den Richtern der Appellationsgerichtshöfe. $\mathrm{Da}$ die Verfassung für die Abwahl aller Richter der oberen Instanzen, d. h. auch für die Appellationsrichter eine Zweidrittel-Mehrheit vorsieht, läßt sich deduzieren, daß auch der Generalkontrolleur nur mit Zweidrittel-Mehrheit abgewählt werden kann.

Außer der allgemeinen Zuständigkeit für die Kontrollaufgaben und der Personalhoheit innerhalb des Rechnungshofes führt die L. O. noch einige weitere für die eigentliche Finanzkontrolle aber unerhebliche Kompetenzen des Generalkontrolleurs an ${ }^{10}$.

\section{Einkünfte und Dispositionsmittel des Generalkontrolleurs}

Das Gehalt des Generalkontrolleurs liegt mit monatlich Q 1300 unter dem Einkommen anderer Spitzenposten in der guatemaltekischen Bürokratie (Staatspräsident Q 6000, Minister im Durchschnitt Q 1500, Parlamentspräsident Q 1500) ${ }^{11}$. In diesem vergleichsweise niedrigen Gehalt kommt das geringe Prestige des Generalkontrolleurs und seiner Behörde zum Ausdruck ${ }^{12}$.

Das offizielle Gehalt des Generalkontrolleurs sagt nichts über seine tatsächlichen Einkünfte aus. Der Staatshaushalt 1973 führt unter der Rubrik „Büro des Chefs“ Q 31968 für Personalkosten auf (Gehälter des Chefs, seines Stellvertreters, der Sekretärinnen und des Chauffeurs). Darüber hinaus stehen dem Generalkontrolleur weitere Q 3600 für Inlandsreisen zur Verfügung; ein Betrag, der angesichts der Größe Guatemalas nur durch eine fieberhafte Reisetätigkeit sachbezogen ausgeschöpft werden könnte. Im Etat des Rechnungshofes findet sich ferner unter der Rubrik "anderweitige Ausgaben“ ein unspezifizierter Globalbetrag von Q 36000 ! Über die Verwendung dieser Summe, die größer ist als der gesamte Personalhaushalt

\footnotetext{
6 Art. 226-228 Constitución de la República

7 Art. 226-228 Constitución de la República

8 Art. 32, Ziff. 1, Art. 62 Regl.

9 Art. 226-228 Constitución de la República

10 So etwa die Gegenzeichnung der gesetzlichen Zahlungsmittel Art. 13 L. O.

11 Die Angaben dieses und des folgenden Abschnittes sind dem Haushaltplan des Rechnungshofes im Staatshaushalt 1973 entnommen.

$12 \mathrm{Im}$ Unierschied hierzu wird der Chef des hochangesehenen Rechnungshofes Costa Ricas wesentlich
} besser als die Minister besoldet. 
des Chefbüros, entscheidet ausschließlich der Generalkontrolleur. Das gleiche gilt für eine weitere Position von jährlich Q 19210 für Inlands- und Auslandsreisen von Mitarbeitern ${ }^{13}$.

Eine gut informierte Quelle ${ }^{14}$ behauptete, der erwähnte Globalposten von Q 36000 für „anderweitige Ausgaben“ werde auch zur Zahlung von Schweigegeldern für übereifrige Bedienstete verwendet. Den nämlichen Zweck erfülle der Etattitel für Reisen von Mitarbeitern. Die Androhung einer Versetzung aus dem Außen- in den Innendienst und der damit verbundene Verlust regelmäßiger Einnahmen aus Reisekosten sei ein wirksames Druckmittel gegen allzu aktive Fahndungsbeamte.

\section{Aufbau und Organisation des Rechnungshofes}

Der Rechnungshof weist drei Abteilungen auf: Die Jefatura - das Büro des Generalkontrolleurs -, das Departamento Administrativo - die Abteilung für Verwaltung - und das Departamento de Auditoría e Investigaciones - die Abteilung für Kontrolle und Recherchen.

5.1 Das Büro des Generalkontrolleurs - die Jefatura

Die Jefatura verfügt über einen nur sehr kleinen Personalstab. Ihm gehören außer dem stellvertretenden Generalkontrolleur nur zwei Sekretärinnen an. Angesichts der Zentralisierung aller wichtigen Entscheidungen in der Person des Generalkontrolleurs garantiert diese personelle Ausstattung ein Maximum an möglicher Diskretion.

5.2 Die Abteilung für Verwaltung - das Departamento Administrativo

Das Departamento ist aufgeteilt in folgende Unterabteilungen:

- Secretaría (Sekretariat)

- Sección de Control de Personal (Personalkontrolle)

- Sección de Archivo de Cuentas (Archivierung v. Rechnungen)

- Sección de Control de Talonarios (Formulare und Rechnungsblöcke)

- Sección de Estadística (Statistik)

- Sección de Servicios (Hausmeister und Bote)

- Sección de Asesoría e Información (Beratung des Generalkontrolleurs)

- Sección de Probidad („Rechtschaffenheit“)

Diese Sektionen erfüllen überwiegend Aufgaben der Innenverwaltung. Für unseren Zusammenhang ist allein die Sección de Probidad von Interesse.

Die Sección de Probidad soll die Anwendung der Ley de Probidad ${ }^{15}$ überwachen.

Die wichtigsten Vorschriften des Gesetzes, dessen Einhaltung einen wesentlichen Beitrag zur Bekämpfung der Korruption leisten könnte, sind: Alle Bediensteten des Staates und der von ihm subventionierten Einrichtungen haben beim Stellenwechsel oder beim Ausscheiden aus dem Dienst eine detaillierte Erklärung über das ihnen und ihren Familienangehörigen eigene Vermögen abzugeben. Diese Erklärungen sollen von einem Kontrolleur des Rechnungshofes innerhalb einer bestimmten

13 Angaben nach dem Haushaltsplan 1973

14 Aus politischen Gründen wird die Quelle nicht bezeichnet.

15 Ley de Probidad, Decreto Presidencial 203. 
Frist geprüft werden. Sofern keine Vergehen vorliegen, wird eine Unbedenklichkeitserklärung erteilt. Die oben erwähnten Personen haben ferner jeweils zu Jahresbeginn eine Vermögenserklärung vorzulegen, sofern im vorausgegangenen Jahr ein Grundstück erworben oder ein Vermögenszuwachs bzw. eine Verschuldung von mehr als Q $1000 \mathrm{zu}$ verzeichnen war. Das Gesetz sieht eine sofortige Entlassung, vorbehaltlich weiterer gerichtlicher Schritte vor, wenn die Vermögenserklärungen Vergehen erkennen lassen oder wenn die Erklärungen nicht abgegeben oder nicht erschöpfend ausgefüllt wurden. Die Bediensteten dürfen außerdem mit der eigenen Behörde keine Geschäfte abschließen. Das Gesetz regelt ferner die Anstellungsvoraussetzungen (z. B. keine Vorstrafen usw.).

Der Sektion sind für die Überwachung der Anwendung der Ley de Probidad u. a. folgende Aufgaben zugewiesen:

Registrierung aller Stellen in den staatlichen Behörden oder in den vom Staat subventionierten Einrichtungen, auf die die Ley de Probidad Anwendung findet, desgleichen Registrierung der Vermögenserklärungen aller Personen, auf die sich die Ley de Probidad bezieht. Sammlung von Informationen über alle Personen, die nach den Vorschriften der Ley de Probidad keine Anstellung finden können. Kontrolle der Einhaltung aller gesetzlichen Normen bei der Abgabe der Vermögenserklärung. Aufforderung an den Generalkontrolleur zur Einleitung von Nachforschungen in Fällen, die dies notwendig erscheinen lassen. Zur Unterstützung der Arbeit der Sektion sind alle Behörden des Staates oder der von ihm subventionierten Einrichtungen verpflichtet. Ernennungen und Entlassungen oder Gründe für Gehaltsänderungen sind der Sektion unverzüglich zu melden.

Wie sich aus dem Haushaltsplan 1973 ergibt, klaffen die gesetzlich vorgeschriebenen Funktionen und die tatsächliche Aufgabenerfüllung weit auseinander. Das gesamte Personal der Sektion besteht aus zwei Schreibkräften (oficinistas) der unteren Kategorie. Die Sektion verfügt weder über den gesetzlich vorgeschriebenen Sektionschef, noch über Planstellen für Beamte des gehobenen und mittleren Dienstes. Die personelle Ausstattung ist daher völlig unzureichend. Schon eine einfache Registratur der Vermögensverhältnisse sämtlicher Bediensteter würde einen wesentlich größeren Apparat voraussetzen. Dies müßte um so mehr der Fall sein, wenn die Sektion ihre Kontrollfunktionen in vollem Umfang wahrnähme.

5.3 Die Abteilung für Kontrolle und Recherchen - das Departamento de Auditoría e Investigación (im folgenden: Dpto. A. e. I.)

Die eigentlichen Aufgaben der Finanzkontrolle des Rechnungshofes werden im Dpto. A. e. I. von drei Sektionen und von Sondereinheiten (Delegaciones speciales) wahrgenommen. Bei den Sektionen handelt es sich um die:

- Sección de Inspecciones y Glosa (Routineüberprüfungen)

- Sección de Auditoría e Investigación (Sonderkontrollen)

- Sección de Juicios de Cuentas (Durchsetzung von Regreßansprüchen)

Im Vergleich zur Jefatura und zum Dpto. Administrativo ist das Dpto. A. e. I. innerhalb des Rechnungshofes bei weitem die größte Abteilung. Dies zeigt der Anteil des Dpto. A. e. I. von Q 948756 am Gesamthaushalt 1973 des Rechnungshofes von Q 12026 786. Für das Büro des Generalkontrolleurs und für die Verwaltungsabteilung werden im Haushalt nur 73 Planstellen ausgewiesen, für das Dpto. A. e. I. hingegen 278 Planstellen. 
Sección de Inspecciones y Glosa ${ }^{16}$

Dieser größten Sektion innerhalb des Dpto. A. e. I., in der die routinemäßig anfallenden Revisionsaufgaben erledigt werden, stehen drei Planstellen für sogenannte Supervisores, 125 Planstellen für Contralores und 16 Planstellen für Bürokräfte des mittleren und unteren Dienstes zur Verfügung. Sie allein verfügt, den Vorschriften entsprechend, über einen Sektionsleiter.

Sección de Auditoría e Investigaciones ${ }^{17}$

In ihre Zuständigkeit fallen alle nicht routinemäßigen Kontrollen. Im Haushaltsplan 1973 sind die Stelle eines Supervisors, 37 Planstellen für Contralores und je zwei Positionen für Bürokräfte ausgewiesen. Da die Sektion mit besonders schwierigen Kontrollaufgaben befaßt ist, werden ihre Mitarbeiter wesentlich besser bezahlt als die der Sección Inspección y Glosa ${ }^{18}$. Der Umstand, daß die Sektion vorschriftswidrig keinen Chef hat, ist darauf zurückzuführen, daß der Generalkontrolleur die Leitung dieser mit politisch heiklen Recherchen beauftragten Unterabteilung sich selbst vorbehält.

Sección de Juicios de Cuentas ${ }^{19}$

Sie ist in erster Linie mit der Durchsetzung der Regreßansprüche beauftragt, die sich aus den von den beiden anderen Sektionen durchgeführten Kontrollen ergeben. Soweit Forderungen den Betrag von Q 10 nicht überschreiten, werden sie bei den Betroffenen geltend gemacht, handelt es sich um höhere Beträge, leitet die Sektion die Angelegenheit innerhalb von 20 Tagen an das Tribunal de Cuentas weiter: ein zweistufiges Sondergericht, das in einem komplizierten Verfahren über Regreßansprüche des Rechnungshofes entscheidet ${ }^{20}$. Zu den Aufgaben der Sektion gehören ferner die Führung einer detaillierten Statistik über Schuldansprüche des Tribunals de Cuentas sowie die Eintragungen dieser Entscheidungen in die Personalakten der Betroffenen. Die Sektion hat außerdem bei den Vermögensuntersuchungen beim Eintritt oder Ausscheiden eines Beamten im Rahmen der Ley de Probidad den zuständigen Stellen die Unterlagen zur Verfügung zu stellen.

Der Personalstab der Sektion ist klein. Nach dem Haushaltsplan 1973 besteht er lediglich aus einem Juristen (Asesor Jurídico) mit zwei Hilfsbeamten sowie drei Bürokräften des mittleren Dienstes.

Aus der Tatsache, daß auch diese Sektion über keinen verantwortlichen Chef verfügt, folgt, daß ihr Personal ebenfalls unmittelbar den Weisungen des Generalkontrolleurs unterworfen ist.

Delegaciones Especiales

Zur Zuständigkeit des Rechnungshofes gehört ferner die Kontrolle autonomer und halbautonomer Institutionen. Sie wird von Sondereinheiten ausgeübt. Nach dem Haushaltsplan 1973 sind 42 Kontrolleure, 6 Hilfsbuchhalter und 6 Bürokräfte des mittleren Dienstes für die Finanzkontrolle von 17 autonomen oder halbautonomen Einrichtungen abgestellt. In einigen Fällen handelt es sich um gut ausgestattete Einheiten ${ }^{21}$. Häufig besteht eine Sondereinheit, aber auch nur aus einem Kontrolleur (so z. B. bei der Bank von Guatemala, beim Nationaltheater und bei der Universität San Carlos).

\footnotetext{
16 Art. 27-31 Regl.

17 Art. 27-31 Regl.

18 Laut Haushaltsplan 1973 beträgt das monatliche Gehalt der Kontrolleure in der Sección A. e. I. Q 390, in der Sección Inspecciónes y Glosa hingegen nur Q 335.

19 Art. 34 Regl.

20 Zum Tribunal de Cuentas Art. 33-112 L. O.

21 Die Delegación Especial, beim Instituto Nacional de Transformación Agraria, die die Staatsgüter
} 
Im Unterschied zu den Secciones de Auditoría e Investigaciones und Inspecciones y Glosa beschränken sich die Sondereinheiten nicht auf eine Kontrolle bereits abgeschlossener Vorgänge. Die permanente Anwesenheit ihrer Beamten in den zu untersuchenden Behörden führt häufig zu einer informellen Vorabklärung der Ausgaben- oder Einnahmenpolitik. Dieses Prüfungsverfahren wird auch bei staatlichen Behörden mehr und mehr angewandt $t^{22}$.

\section{Die Rechnungsprïfer - Contralores de Cuentas}

Die eigentlichen Kontrollaufgaben werden von den Rechnungsprüfern, den Contralores de Cuentas ausgeübt. Eingangsvoraussetzungen sind u. a. eine abgeschlossene Ausbildung als Buchhalter, ersatzweise eine fünfjährige Buchhalter- bzw. zehnjährige Erfahrung in Spezialbereichen des Steuer- und Buchhaltungswesens ${ }^{23}$.

Nach dem Gesetz über den Rechnungshof haben alle Kontrolleure den gleichen Rang, die gleichen Rechte und die gleichen Verpflichtungen ${ }^{24}$. Das Gesetz fordert ferner, daß die Rechnungsprüfer ihre Aufgaben in absoluter Unabhängiglseit wahrnehmen und "nicht das Objekt von Repressalien oder Belästigungen bei der legitimen Ausübung ihrer Pflichten sein sollen"25 ". Alle Bediensteten des Staates und der von ihm subventionierten Einrichtungen werden verpflichtet, ihre Bücher und sonstige Unterlagen den Prüfern auszuhändigen und ihnen jede gewünschte Information zu erteilen ${ }^{26}$. Die Rechnungsprüfer können Beamte auf Zeit vom Dienst suspendieren ${ }^{27}$. Die Eigeninitiative der Rechnungsprüfer wird allerdings durch die Durchführungsverordnung erheblich eingeschränkt. Dort heißt es lapidar, daß der Rechnungsprüfer die Aufgaben auszuführen habe, die ihm vom Generalkontrolleur oder vom Sektionschef aufgetragen werden ${ }^{28}$. Es kommt hinzu, daß die Prüfer für Fehler oder Kompetenzüberschreitungen haftbar gemacht werden können. Die Behörde leistet keinen Schadenersatz ${ }^{29}$.

Die Rechnungsprüfung selbst wird vom Gesetz über den Rechnungshof und von der Durchführungsverordnung bis in völlig unwichtige Details hinein peinlich genau geregelt.

\section{Würdigung der Rolle des Rechnungshofes}

In einer 1967 der Universität San Carlos vorgelegten Dissertation wird über den Rechnungshof ausgeführt ${ }^{30}$ :

„Man hat uns informiert, daß ein großer Teil des Personals des Rechnungshofes aus politischen Gründen oder auf Grund freundschaftlicher Beziehungen

verwaltet (meistens handelt es sich um im Krieg enteignete Kaffeeplantagen vormals deutscher Besitzer)

besteht z. B. aus einem Chef, zehn Kontrolleuren und drei Bürokräften; die Delegación Especial bei der Verwaltung der Stadt Guatemala weist einen Chef, drei Kontrolleure und drei Hilfsbuchhalter auf.

22 Gespräch mit dem Generalkontrolleur am 27. 3. 1973. So sind z. B. bei der D. G. Aduanas mehrere Rechnungshofsbedienstete tätig. Auch dem Flughafenzollamt sind mehrere Beamte zugeteilt. Nach Auskunft des Generalkontrolleurs haben sich diese Maßnahmen als sehr erfolgreich erwiesen.

23 Art. 10. L. O.

24 Art. 11 L. O.

25 Art. 28 L. O.

26 Art. 19 L. O.

27 Art. 12 Ziff. 18 L. O., Art. 62 Regl.

28 Art. 32 Ziff. 1, Art. 62 Regl.

29 Art. 33 Regl.

30 Zitate aus Leon Peláez, Julio Cesar: Organismos Fiscalizadores en Guatemala (Tesis) Guatemala 1967, S. $50 \mathrm{f}$. 
eingestellt wurde, ohne daß die unverzichtbaren Minimalerfordernisse erfüllt waren, die den modernen Techniken der Personalauswahl entsprechen. Das logische Resultat ist, daß von Leuten, die unter solchen Umständen ernannt wurden, weder Effizienz noch Moral verlangt werden kann ... Unter den Personen, die im Rechnungshof arbeiten, findet sich ein einziger Akademiker mit Studienabschluß; obwohl einige gerade ihr Studium abschließen . . ., sind sie eine bemerkenswerte Minderheit, was angesichts des Zuständigkeitsbereichs des Rechnungshofes nicht zu rechtfertigen ist. Deshalb und wegen der Kompliziertheit der Recherchen, wegen der mangelnden Übersicht und der vollkommenen Ignoran'z der Kontrolleure sind die Rechnungsprüfer in vielen Fällen außenstehenden Spezialisten bei wichtigenAngelegenheiten unterlegen."

Der Verfasser gibt der Hoffnung Ausdruck, daß sich durch den Eintritt von Universitätsstudenten oder Absolventen die von ihm beschriebene Situation ändern könne, ja sich bereits zu wandeln beginne.

Die Berufsausbildung der Kontrolleure scheint sich tatsächlich gebessert zu haben. Der derzeitige Generalkontrolleur bemüht sich darum, die Funktionsfähigkeit des Rechnungshofes durch bessere Ausbildung des Personals und durch Rationalisierung der Arbeitsvorgänge zu verbessern ${ }^{31}$. $\mathrm{Zu}$ erwähnen ist ferner, daß von ihm seit einigen Jahren eine Zeitschrift herausgegeben wird, in der laufend für die Arbeit des Rechnungshofes wichtige Gesetzestexte veröffentlicht werden ${ }^{32}$.

Es kann jedoch nicht die Rede davon sein, daß bei der Einstellung der Kontrolleure und bei ihren Amtshandlungen die gesetzlichen Normen stets eingehalten werden. Nicht selten werden gänzlich ungeeignete Bewerber ernannt. Oftmals sind gute Beziehungen wichtiger als ausreichende Qualifikationen. Wirklich fähige Personen ziehen eine Tätigkeit in der Privatwirtschaft vor.

Die Bestimmung, bei der Wahrnehmung ihrer Pflichten dürften gegen Kontrolleure keine Repressalien ausgeübt werden, liegt quer zur politischen Realität Guatemalas. Ein gut informierter Kenner der Verhältnisse im Rechnungshof hat das Leben der Kontrolleure als „via crucis" bezeichnet ${ }^{33}$. Bei den Versuchen, Unterschlagungen oder andere finanzielle Delikte einflußreicher Personen aufzudecken, muß der recherchierende Kontrolleur mit schweren Nachteilen, bis hin zur Bedrohung seiner physischen Existenz, rechnen.

Wie die Analyse der Organisation des Rechnungshofes und seines Budgets zeigten, finden sich an allen Nervenpunkten des Überwachungssystems bemerkenswerte Mängel, die sich kaum als bloße Nachlässigkeit erklären lassen. Die gilt insbesondere für die Funktionsunfähigkeit der Sección de Probidad und für das Fehlen der vorgeschriebenen Stellen eines Chefs in den Secciones de Auditoría e Investigaciones und Juicios de Cuentas. Die Folge dieser Etatpolitik ist, daß alle wichtigen Kontrollpositionen in der Hand des Generalkontrolleurs vereinigt sind. Auf diese Weise ist dafür gesorgt, daß wichtige Fahndungen nur anlaufen können, wenn dies dem Generalkontrolleur opportun erscheint. Es ist ferner bemerkenswert, daß ausgerechnet der Chef des Rechnungshofes über einen für guatemaltekische Verhältnisse gut ausgestatteten Dispositionsfonds frei verfügen kann.

Die Unabhängigkeit des Generalkontrolleurs gegenüber politischen Instanzen ist auch angesichts seiner relativ kurzen Amtszeit von vier Jahren begrenzt. Es

31 Dieser Eindruck ergab sich u. a. aus einem Gespräch mit dem Generalkontrolleur am 27. 3. 1973.

32 Revista Informativa, Contraloría de Cuentas, Guatemala, Nr. 1 Sept. 1971, Nr. 2 Dez. 1971, Nr. 3,4,5 Sept. 1972.

33 Die Quelle kann aus politischen Gründen nicht genannt werden. 
kommt hinzu, daß er von der Regierungsmehrheit im Parlament gewählt wird. Im Rahmen der politischen Verhältnisse Guatemalas ist sein Handlungsspielraum gegenüber der Exekutive sehr eingeschränkt. Dies um so mehr, als der Gesetzgeber - im Unterschied zur Rechtslage in Costa Rica - keine Vorschriften erlassen hat, wonach verwandtschaftliche Beziehungen zu hochgestellten Personen der Exekutive die Berufung zum Generalkontrolleur ausschließen. Somit stehen dem Umstand, daß der amtierende Generalkontrolleur zur Zeit seiner Wahl mit dem Präsidenten Guatemalas verschwägert war, keine rechtlichen Hindernisse im Wege.

Das Gesetz über den Rechnungshof verpflichtet den Generalkontrolleur, staatlichen Stellen jederzeit Auskunft über die in seinem Haus befindlichen Informationen zu erteilen, und nach Anforderung für staatliche Organe Expertisen anzufertigen sowie Fahndungen und Buchprüfungen einzuleiten ${ }^{34}$. Diese Bestimmungen und die mit ihnen verbundene Praxis haben dem Rechnungshof den Vorwurf eingebracht, er sei primär eine politische Waffe der Regierung zur Unterdrückung der Opposition. Vor allem am Vorabend von Wahlen werde der Rechnungshof gegen Politiker und Beamte der Opposition zur Überprüfung ihrer finanziellen Aktivitäten ins Feld gerufen. Angesichts der auch in den Reihen der Oppositionspolitiker weitverbreiteten Korruption, habe er sich in dieser Funktion sehr „bewährt“. Die Regierung könne auf diese Weise durch den Rechnungshof lästige Gegner in den Kommunen oder in den autonomen und halbautonomen Institutionen ausschalten $^{35}$.

\section{Der Rechnungshof Costa Ricas (Contraloría General de la República)}

\section{Rechtliche Grundlagen}

Die staatsrechtliche Stellung, die Kompetenzen und der organisatorische Aufbau des Rechnungshofes werden in Artikel 183 und 184 der Verfassung sowie im Gesetz über den Rechnungshof ${ }^{36}$ und in einer Durchführungsverordnung, dem Reglamento de la Contraloría General ${ }^{37}$, geregelt. Die Bestimmungen der Durchführungsverordnung wurden allerdings in einigen unwichtigen Punkten nicht verwirklicht oder durch die Wirklichkeit überholt ${ }^{38}$.

\section{Verfassungsrechtliche Stellung}

Der Rechnungshof ist als Hilfsorgan der Legislative bestellt. Die Verfassung gewährt ihm „absolute Unabhängigkeit bei der Wahrnehmung seiner Funktion“ und der eigenen Verwaltung ${ }^{39}$. Die Verfassungsväter wollten mit ihm dem Parlament ein effektives Instrument zur Kontrolle der staatlichen Finanzen in die Hand geben, dessen Unabhängigkeit aber auch gegenüber der Legislative gewahrt bleiben sollte. Wegen der starken politischen Stellung des Rechnungshofes in Haushaltsangele-

\footnotetext{
34 Art. 14 L. O.

35 So nach den Angaben verschiedener Beamten und Politiker.

36 Ley Orgánica de la Contraloría General, Decreto No. 1252, 23. 12. 1950 im folgenden zitiert L. O.

37 Decreto No. 9, 29. 3. 1962, im folgenden zitiert Regl.

38 Dies gilt insbesondere für die Organisationsstruktur des Rechnungshofes.

39 Art. 183 Constitución.
} 
genheiten, die die Regierung zwingt, seine Reaktionen möglichst $\mathrm{zu}$ antizipieren, hat sich in der Praxis ein gutes Kooperationsverhältnis zwischen dem Rechnungshof und der Exekutive entwickelt. Für das Verhältnis zur Exekutive ist von Bedeutung, daß der Leiter und Stellvertreter des Rechnungshofes vom Parlament gewählt werden; dabei kann die Regierungspartei ihren Einfluß geltend machen. Dies heißt jedoch nicht, daß der der Regierung genehmste Kandidat gewählt wird. Ahnnlich wie im politischen System der Vereinigten Staaten und im Unterschied zu den meisten parlamentarischen Systemen stellt die Legislative in der Präsidialdemokratie Costa Ricas eine eigenständige, die Regierung kontrollierende Kraft dar. Das wirkt sich auch auf die Stellung ihres Hilfsorgans, des Rechnungshofes aus.

\section{Kompetenzen}

Der Rechnungshof ist für die Kontrolle der staatlichen Einnahmen und Ausgaben zuständig. Darüber hinaus nimmt er neben vielfältigen anderen Aufgaben entscheidenden Einfluß auf die Verabschiedung des Staatshaushaltes. Diese zentralen Funktionen überlappen sich naturgemäß an verschiedenen Stellen.

\subsection{Finanzkontrolle}

Der Rechnungshof überprüft alle staatlichen, kommunalen oder autonomen Behörden einschließlich ihrer Bediensteten, sofern sie in irgendeiner Weise öffentliche Gelder oder öffentliches Eigentum verwalten ${ }^{40}$. Er überwacht ihr Finanzverhalten sowohl unter rechnerischen Gesichtspunkten wie auch unter dem Aspekt der kostengünstigen und sachadäquaten $Z$ weckerfüllung. Seine Kompetenzen beschränken sich nicht nur auf die Kontrolle bereits abgeschlossener Vorgänge. So hat er z. B. die Rechnungen aller staatlichen und kommunalen Behörden und der mit öffentlichen Geldern subventionierten Einrichtungen im voraus zu überprüfen, d. h. jede Zahlungsanweisung wird erst nach vorheriger Prüfung und Unbedenklichkeitserklärung durch den Rechnungshof wirksam ${ }^{41}$. Die betroffenen Behörden und Personen sind verpflichtet, der Contraloría bei der Durchführung ihrer Aufgaben alle gewünschten Informationen und sonstigen Hilfen zu geben ${ }^{42}$. Die Verweigerung solcher Unterstützung kann auf Antrag des Leiters des Rechnungshofes mit der sofortigen Suspendierung oder Entlassung der betreffenden Person geahndet werden ${ }^{43}$. Bei Verstößen gegen finanzielle Interessen des Staates kann der Generalkontrolleur auf dem Wege über den Generalstaatsanwalt ein Strafverfahren einleiten lassen ${ }^{44}$.

\subsection{Einwirkung auf öffentliche Haushalte}

Die Contraloría hat das Parlament in allen Haushaltsfragen zu beraten ${ }^{45}$. Die Verfassung sieht vor, daß Erhöhungen des von der Regierung vorgelegten Budgets von der Legislative nur nach vorangegangener Konsultation des Rechnungshofes

\footnotetext{
40 Art. 3 L. O.

41 Art. 4 Abs. i L. O.

42 Art. 23 L. O.

43 Art. 7 Abs.

44 Art. 21 L. O.

45 Art. 4 Abs. b L. O
} 
über die Deckungsmöglichkeiten beschlossen werden dürfen ${ }^{46}$. Darüber hinaus hat der Gesetzgeber den Rechnungshof ermächtigt, die Haushaltsrechnung zu überprüfen ${ }^{47}$. Die Regierung hat ihm diese jeweils bis zum 1. März zu übermitteln; der Rechnungshof wiederum fertigt darüber ein detailliertes Gutachten an, auf Grund dessen das Parlament über die Entlastung der Regierung entscheidet. Außerdem hat die Contraloría einen jährlichen Tätigkeitsbericht vorzulegen, in dem sie über das wirtschaftliche Ergebnis des vorausgehenden Finanzjahres berichtet und dem Parlament Empfehlungen zu Fragen der künftigen Haushaltsgestaltung zuleitet ${ }^{48}$. Der Rechnungshof muß darüber hinaus monatlich im Amtsblatt (Diario Official) detailliert über den jeweiligen Stand der staatlichen Finanzen berichten ${ }^{49}$. Was die Kommunen und autonomen Einrichtungen betrifft, so stehen dem Rechnungshof weitreichende Aufsichtsfunktionen zu. Gemeindeabgaben und die Haushalte der Kommunen und autonomen Einrichtungen bedürfen der Prüfung und Genehmigung durch den Rechnungshof ${ }^{50}$.

\subsection{Weitere Funktionen}

Dazu zählen die Registrierung aller Gesetze, Verordnungen und Verträge des Staates, mit denen finanzielle Verpflichtungen verbunden sind ${ }^{51}$, die Genehmigung und Kontrolle der Rechnungsformulare sowie die Überwachung der Rechnungsbücher und des Buchhaltungsverfahrens ${ }^{52}$.

Der Rechnungshof entscheidet über Einsprüche gegen Beanstandungen durch die "Contabilidad Nacional53", er hat außerdem alle von Notaren und Wirtschaftsprüfern ausgefertigten Beglaubigungen und Verträge $\mathrm{zu}$ archivieren, aus denen sich Ansprüche des Staates herleiten lassen ${ }^{54}$.

\section{Leitung des Rechnungshofes}

Der Rechnungshof wird vom Generalkontrolleur (Contralor General), in seiner Abwesenheit vom Sub-Contralor, geleitet.

Der Generalkontrolleur und sein Stellvertreter werden vom Parlament für eine Amtsdauer von 8 Jahren gewählt; eine Wiederwahl ist möglich ${ }^{55}$. Der Generalkontrolleur und sein Stellvertreter können von der Legislative nur mit einer Zweidrittel-Mehrheit der Mitglieder abgesetzt werden ${ }^{56}$. Der Generalkontrolleur übt die Personalhoheit über die Bediensteten seiner Behörde aus ${ }^{57}$. Alle diese Bestimmungen - , die ungewöhnlich lange Amtszeit, die Erschwerung der Abwahl durch ein Zweidrittel-Quorum sowie die Personalhoheit, die eine Einwirkung der Regierung und des Parlaments auf die Personalpolitik innerhalb des Rechnungshofes sehr erschwert - bilden das Fundament der Autonomie der Contraloría.

46 Art. 179 Constitución.

47 Zum folgenden vgl. Art. 181 Constitución.

48 Art. 17 L. O.

49 Art. 18 L. O.

50 Art. 4 Abs. i L. O.

51 Art. 4 Abs. g L. O

52 Art. 8 Abs. c L. O.

53 Art. 4 Abs. e L. O.

54 Art. 25 L. O.

55 Art. 183 Constitución.

56 Art. 183 Constitución.

57 Art. 183 Constitución und Art. 14 L. O. 
Das Gesetz über den Rechnungshof enthält verschiedene Vorschriften, die einen Mißbrauch der weitreichenden Vollmachten des Generalkontrolleurs verhindern sollen. Dem Generalkontrolleur und Subkontrolleur sind politische Aktivität, Ausübung eines Nebenberufs und Übernahme sonstiger öffentlicher Ämter untersagt ${ }^{58}$. Besonders aufschlußreich sind die detaillierten gesetzlichen Bestimmungen zur Verhinderung von Nepotismus ${ }^{59}$. So dürfen der Generalkontrolleur und sein Vertreter mit dem Präsidenten, den Vizepräsidenten, den Ministern und den Direktoren der autonomen Institutionen weder in direkter Linie noch in der Seitenlinie (jeweils bis ins dritte Glied) verwandt oder verschwägert sein. Dem Generalkontrolleur und Subkontrolleur wird die Einflußnahme auf Angelegenheiten verboten, an denen sie selbst und ihre Angehörigen (im obigen Sinne) direkte oder indirekte Interessen haben. Weitreichend ist die Bestimmung, daß nicht nur die Einstellung von Angehörigen der beiden Spitzenbeamten, sondern auch die Ernennung von Angehörigen aller anderen Mitarbeiter untersagt ist ${ }^{60}$.

Diese sehr konkreten Vorschriften sind angesichts einer kulturellen Tradition, die den Nepotismus begünstigt, sehr sinnvoll. Sie sind nicht bloßer gesetzlicher Anspruch geblieben, sondern in die Wirklichkeit umgesetzt worden.

Eine Entlassung von Bediensteten des Rechnungshofes ist nur bei schweren Vergehen (z. B. Bestechung) möglich. Ämter- und Laufbahnpatronage werden durch strenge Aufnahme (vgl. 6. Personal) - und Laufbahnbestimmungen in weitem Umfang ausgeschlossen. Während im Rechnungshof Guatemalas die meisten personalpolitischen Entscheidungen vom Generalkontrolleur im Alleingang entschieden werden, ist im Rechnungshof Costa Ricas die Personalpolitik dezentralisiert. Bei Einstellungen oder Beförderungen müssen der Chef des Personalbüros und die Direktoren der Abteilung gehört werden.

\section{Organisationsstruktur}

5.1 Zur Innenverwaltung des Rechnungshofes gehören das Büro des Generalkontrolleurs, eine Verwaltunggseinheit für Dienstleistungen (Hausmeister, Chauffeure, Wächter etc.) und ein Personalbüro (Oficina de Personal).

5.2 Die Außenverwaltung obliegt den folgenden sieben Departamentos:

- Departamento de Estudios Económicos (Finanzwissenschaftliche Analysen und Archivierung)

- Departamento de Estudios Especiales (Sondergutachten zu schwierigeren Fragen der Haushalts- und Finanzkontrolle)

- Departamento de Ingeniería (technische Kontrolle der staatlichen Beschaffungspraxis)

- Departamento de Auditoría (Finanzkontrolle)

- Departamento de Licitaciones (Kontrolle der Beschaffungspraxis)

- Departamento Control de Presupuestos (Haushaltskontrolle)

58 Art. 13 und Art. 12 a L. O.

59 zum folgenden vgl. Art. 12 Abs. b und Art. 14 L. O. 60 Art. 14 L. O. 
Alle Departamentos sind personell gut ausgestattet. Sie werden jeweils von einem ranggleichen Direktor geleitet. Die starke Stellung der Direktoren bedingt in der Praxis ein erhebliches Maß innerorganisatorischer Gewaltenteilung und Kontrolle. Das Departamento de Auditoría ist zuständig für die Prüfung der Bücher und Rechnungen aller staatlichen, kommunalen und autonomen Einrichtungen ${ }^{61}$. Wegen des Umfanges ihrer Aufgaben ist diese Abteilung mit 98 von insgesamt 236 Planstellen, die bei weitem größte Verwaltungseinheit ${ }^{62}$. Sie ist in drei Unterabteilungen (Secciones) zur Kontrolle staatlicher, kommunaler und autonomer Einrichtungen gegliedert. Das Departanento de Licitaciones überwacht die Beschaffung der öffentlichen Hand in rechnerischer und sachlicher Hinsicht ${ }^{63}$. Es wird in technischen Angelegenheiten (z. B. Materialqualität) vom Departamento de Ingeniería, in rechtlichen Fragen vom Departamento Legal unterstützt. Die übrigen Abteilungen widmen sich der Haushaltskontrolle.

\section{Personal}

Die Bediensteten werden aufgrund einer anspruchsvollen Eignungsprüfung eingestellt. Sie ist vor einer Kommission abzulegen, der der Personalchef, der Leiter der Rechtsabteilung und der Chef der betreffenden Abteilung angehören ${ }^{64}$. Eine Ausnahme gilt für die Angehörigen bestimmter Berufsgruppen (Rechtsanwälte, Ingenieure). Bei ihnen wird dem Bewerber mit dem besten beruflichen Leistungsnachweis der Vorzug gegeben ${ }^{65}$.

\section{Würdigung}

Der Rechnungshof genießt im Lande ein hohes Ansehen. Seiner Unabhängigkeit und Effizienz ist eine nicht nur für Lateinamerika vorbildliche Kontrolle der öffentlichen Finanzen zu verdanken. Bezeichnend für die Sorgfalt des Gesetzgebers bei dem Bemühen, spezifisch lateinamerikanische Korruptionsursachen zu bekämpfen, sind die Bestimmungen zur Verhinderung von Nepotismus. Charakteristisch für die politische Kultur Costa Ricas ist außerdem die Tatsache, daß die politischen Kräfte des Landes bei den bisherigen Wahlen des Generalkontrolleurs und seines Stellvertreters zumindest bemüht waren, einen möglichst breiten politischen Konsens herzustellen.

Kritisch ist $\mathrm{zu}$ vermerken, daß es sich bei dem Rechnungshof um eine überaus kostspielige Superkontrollbehörde handelt. So entsprach der Personaletat des Rechnungshofes 1973 mit C 7.673.580 dem des Außenministeriums einschließlich aller diplomatischen Vertretungen mit C 7.439.641. Er lag ferner beträchtlich über dem der Ministerien für Wirtschaft und Arbeit. In der Abteilung für Rechnungsprüfung waren 1973 ca. 90 Rechnungsprüfer tätig. Demgegenüber sind im Haushalt Baden-Württembergs von 1974 für den Rechnungshof nur 60 Rech-

\footnotetext{
61 Art. 60 Regl.

62 Nach dem Staatshaushalt 1973. Nach ihm ist die zweitgrößte Abteilung das Dpto. Control de Presupuestos mit 52 Planstellen. Das Dpto. Estudios Economicos hat 17, das Dpto. Licitaciones 13 und das Dpto. Legal 12 Planstellen. Die übrigen Dptos haben jeweils weniger als 10 Planstellen.

63 Art. 66 Regl.

64 Art. 18 Regl.

65 Art. 18 Regl.
} 
nungsräte ausgewiesen. Hierbei ist im Vergleich zu Costa Rica ein sehr viel größeres und komplexeres Prüfungsvolumen zu bewältigen. Bei aller Wirksamkeit seiner Kontrollmaßnahmen läßt sich nicht übersehen, daß durch sie nicht selten der staatliche Funktionsablauf beeinträchtigt wird. So hat z. B. die gesetzliche Regelung, daß jede Rechnung vor Bezahlung rechnerisch und sachlich vom Rechnungshof $\mathrm{zu}$ prüfen ist, eine erhebliche Verzögerung des Zahlungsverkehrs zur Folge. Dies um so mehr, als auch die kleinste Rechnung nicht nur die Unterschrift des Generalkontrolleurs aufweisen, sondern außerdem von mehreren anderen Behördenchefs (z. B. Finanzminister, zuständiger Ressortminister, Chef der Tesorería Nacional etc.) abgezeichnet werden muß. Kenner der Situation meinen, daß die vom Rechnungshof ausgeübte Kontrolle der öffentlichen Hand in verschiedenen Bereichen wesentlich kostengünstiger durch technische Verbesserungen des Buchhaltungswesens erreicht werden könnte.

\section{Zusammenfassender Vergleich}

Bei einem Vergleich der beiden Rechnungshöfe ergibt sich folgender Befund:

1. Im Rechnungshof Guatemalas werden alle wichtigen Entscheidungen vom Generalkontrolleur selbst getroffen. Eine interne Überwachung oder Einschränkung seiner Leitungsbefugnisse gibt es so gut wie nicht. Demgegenüber wird die Entscheidungskompetenz des Generalkontrolleurs Costa Ricas durch ein beträchtliches Maß innerorganisatorischer Gewaltenteilung und durch die beamtenrechtliche Absicherung der Bediensteten erheblich eingeschränkt.

2. Der Generalkontrolleur Costa Ricas wird für acht Jahre, der Generalkontrolleur Guatemalas nur für vier Jahre gewählt, wobei in beiden Fällen eine Wiederwahl zulässig ist. Daraus ergibt sich, daß die Unabhängigkeit des Generalkontrolleurs Guatemalas gegenüber politischen Pressionen sehr viel geringer ist.

3. Während in Costa Rica Gesetz und Gesetzespraxis zur Deckung gebracht werden, klaffen sie in Guatemala in vielen Fällen weit auseinander. So steht beispielsweise die Ley de Probidad nur auf dem Papier. Ihre Überwachung durch den Rechnungshof findet nicht statt. Die gesetzlich vorgeschriebene Organisationsstruktur ist im Hinblick auf die Vakanzen in wichtigen Uberwachungspositionen (Sektionsleiter) nur sehr unvollständig verwirklicht. Der gesetzlich geforderte Schutz der Rechnungsprüfer gegen organisationsinterne und gegen externe Pressionen ist nicht gewährleistet.

4. Die für den Rechnungshof Costa Ricas gültigen gesetzlichen Bestimmungen gegen Nepotismus tragen der soziokulturellen Tradition Lateinamerikas Rechnung. Demgegenüber ist es für Guatemala bezeichnend, daß sich hier derartige gesetzliche Regelungen nicht finden. Charakteristisch in diesem Zusammenhang erscheint es ferner, daß der derzeitige Generalkontrolleur bei seiner Wahl mit dem amtierenden Staatspräsidenten verschwägert war. 
5. Trotz eines relativ guten Kooperationsverhältnisses zur Regierung hat sich der Rechnungshof Costa Ricas als Kontrollorgan der Exelutive bewährt. Dagegen blieb der Rechnungshof Guatemalas auch nach der 1965 geänderten Bestellungspraxis für das Amt des Generalkontrolleurs (nicht mehr Ernennung durch den Präsidenten, sondern Wahl durch das Parlament) ein weitgehend im Interesse der Regierung und ihrer Anhänger handelndes Organ. Die Vorwürfe, der Rechnungshof habe eine Alibifunktion für die Bemäntelung der Korruption der herrschenden Elite, und er werde gleichzeitig zur Bekämpfung der politischen Opposition eingesetzt, scheinen nicht unbegründet zu sein.

6. Die öffentliche Verwaltung Costa Ricas ist in ganz Lateinamerika zu Recht wegen ihrer geringen Korruptionsanfälligkeit bekannt. Demgegenüber sagt man wohl ebenfalls zu Recht - der guatemaltekischen staatlichen Verwaltung nach, $\mathrm{da} ß$ auf sie die gängigen Vorstellungen über Korruption in den Bürokratien armer Entwicklungsländer anwendbar seien.

Angesichts der dezidierten Unterschiede in der Korruptionsanfälligkeit der Bürokratien beider Staaten stellt sich die Frage nach der Bedeutung der Rechnungshöfe für die Bekümpfung von Korruption. Die entscheidenden Ursachen für Erfolge bei der Bekämpfung von Korruption dürften hierbei weniger in der Existenz der Rechnungshöfe selbst oder in rechtlich-administrativen Details ihrer Struktur, als vielmehr in den allgemeinen konstitutiven politischen Rahmenbedingungen enthalten sein. Die Funktionsfähigkeit des Rechnungshofes Costa Ricas als Einrichtung der präventiven Korruptionsbekämpfung ist primär in dem freiheitlichen politischen System und der politischen Kultur Costa Ricas begründet. Die Leistungsfähigkeit des Rechnungshofes wird nicht nur durch ein funktionierendes Gewaltenteilungs- und Parteiensystem, sondern vor allem auch durch eine wache, politisch aktive Offentlichkeit geschützt. Besonders bemerkenswert ist das hohe Niveau der Presse. Umgekehrt spricht viel dafür, daß die oben skizzierten Mängel des guatemaltekischen Rechnungshofes letzten Endes ihren Grund in Mängeln des politischen Systems haben. In Guatemala, einem Land, in dem die parlamentarischen demokratischen Institutionen nur die Fassade für die Herrschaft einer kleinen Minderheit bilden, in dem die politischen Sprecher der parlamentarischen Opposition in mannigfaltiger Weise terrorisiert werden, eine freie Berichterstattung in der Presse unterdrückt wird und die politische Herrschaft sich auf ein weitverzweigtes System der Korruption stützt, müssen allen Versuchen der Bekämpfung öffentlicher Korruption sehr enge Grenzen gezogen sein. 
training of its members. In the third part these features are interpreted. Next the modalities of foundation, the organs of a social property firm and of the social property sector as well as the financial system are analysed. Finally the intended objectives of the Propiedad Social are compared with their specification in the law and with the possibility of implementation.

Besides that the first projects of the Propiedad Social are explained.

The analysis of the social property legislation leads to the following conclusions:

- Growth and expansion of a social property firm have priority over self-management and participation.

- Neither in conception nor in practical organisation the participation of the members of the social property firm in management has been guaranteed sufficiently in the law.

- There is a risk of concentration of entrepreneurial decisions in a technical and administrative elite.

- The governmental representatives dominate the highest organs of the sector, and the most important decisions concerning the existence of the social property firm have to be approbated by the Comisión Nacional de Propiedad Social. Therefore in the social property there may be a nucleus for a new form of state capitalism.

- In the law have not been established any mechanisms by which the workers' self-management discharges into a political participation as called by the government.

\section{Auditing Boards in Guatemala and Costa Rica}

\section{By Dieter Oberndörfer}

The article investigates legal provisions for, and organizational structures of, the Auditing Boards in Guatemala and Costa Rica.

Its essential conclusions are summarized as follows:

1. In Guatemala all decisions of consequence are made by the Principial Auditor himseif. Internal supervision or restriction of his authority is practically nonexistent. In Costa Rica, on the other hand, the authority of the Principal Auditor is restricted by a considerable degree of internal division of competence and by a Civil Service codex providing protection for officials.

2. The term of office is 8 years in Guatemala; 4 years however in Costa Rica, and in both cases re-election is possible. As a result the independence of the Principal Auditor in Guatemala in the face of political pressure may be far more restricted.

3. Whilst in Costa Rica law and practice agree, these show a wide discrepancy in Guatemala. The statutory supervision of the Ley de Probidad by the Auditing Board for instance is non-existent in many instances.

4. It is significant that in Guatemala - other than in Costa Rica - there is no legal provision against nepotism.

5. Despite a relatively good cooperation with the government the Auditing Board in Costa Rica has proved itself an effective control of the executive. In contrast, 
the Guatemalan Auditing Board continued to be a body acting largely in the interests of the government and its followers.

6. The fact that the Costa Rican Auditing Board has proved itself an efficient instrument in the prevention of corruption is due primarily to the liberal political system and cultural climate of the country. Its effectiveness is maintained not alone by the prevailing political party system and the constitutional separation of power but foremost by a critical and politically active public opinion. Notable in this connection is the high standard of the press. By contrast, it appears that deficiencies as apparent in the Auditing Board of Guatemala can largely be traced back to the deficiencies in the country's political system.

\section{Law and Politics in Revolutionary-Ethiopia}

\section{By Heinrich Scholler und Paul Brietzke}

Ethiopia's "creeping revolution", when analyzed in stages, illuminates the interrelation of law and politics where direct military intervention occurs in African political life and the tensions between a traditional society and a modernizing urban elite, between the assertion of political and economic rights and between socialist legal values and the rule of law. Ethiopian public law had hitherto conferred broad discretionary authority and consolidated the power of the Amhara-dominated Monarchy, feudality, bureaucracy and the Orthodox Church. Maladministration and civilian paralysis in the face of mounting socio-economic crises could not be averted and in one year, the Military was able to consolidate power and shift its policy emphasis from progress towards popular government to the implementation of socialist economic policies. The 1974 Draft Constitution, embodying Western jural postulates, was rejected because it did not sufficiently embrace an evolving nationalist-socialist ideology. In its place, the emerging "constitutional law" of the new Proclamations gives the Military's representatives concentrated and almost unlimited power - an accurate reflection of political realities.

Ethiopia is also experiencing a period of revolutionary justice; an attempt to increase the speed and certainty of conviction of "enemies of the people", and the fostering of the Military's political stability and legitimacy and socio-economic change through the coercive use of penal law. New penal laws, for the most part, merely restate Penal Code provisions while radically increasing penalties, altough a few new, broad and retroactive offenses have been created. The use of law in revolutionary Ethiopia shows a recognition of the need for administrative continuity and the development of an alternate rule system that embodies the revolutionary ethos. Western legal theory provides imperfect responses to the challenges posed by these revolutionary uses of law, particularly in Ethiopia. 